\title{
Single shot Kerr magnetometer for observing real-time domain wall motion in permalloy nanowires
}

\author{
P Möhrke ${ }^{1}$, T A Moore ${ }^{1}$, M Kläui ${ }^{1}$, J Boneberg ${ }^{1}$, D Backes $^{1,2}$, S Krzyk ${ }^{1}$, \\ L J Heyderman ${ }^{2}$, P Leiderer ${ }^{1}$ and U Rüdiger ${ }^{1}$ \\ ${ }^{1}$ Fachbereich Physik, Universität Konstanz, Universitätsstr. 10, 78457 Konstanz, Germany \\ ${ }^{2}$ Laboratory for Micro- and Nanotechnology, Paul Scherrer Institut, 5232 Villigen, Switzerland \\ E-mail: Mathias.Klaeui@uni-konstanz.de
}

Published 1 August 2008

\begin{abstract}
Details are presented of a single shot focused magneto-optic Kerr effect (MOKE)

magnetometer which is used to capture the movement of single domain walls (DWs) in permalloy $\left(\mathrm{Ni}_{80} \mathrm{Fe}_{20}\right)$ nanowires ( $\geqslant 400 \mathrm{~nm}$ width and $\geqslant 20 \mathrm{~nm}$ thickness) in real time. By probing the DW motion within the $1 \mu \mathrm{m}$ diameter laser spot of the instrument, DW velocity and pinning field distributions were obtained. An external field was ramped up linearly, and depinning of a DW from the same start position was observed at three different fields, indicating the stochastic nature of the DW motion.
\end{abstract}

(Some figures in this article are in colour only in the electronic version)

\section{Introduction}

The motion of domain walls (DWs) in magnetic nanowires is of strong fundamental and technological interest. Nanometresized magnetic structures can serve as ideal model systems for exploring the dynamics of DWs induced by external magnetic fields or spin-polarized currents [1-3] and offer the prospect of new spintronic devices for data storage or logic applications $[4,5]$.

Since the magnetization direction in permalloy $(\mathrm{Py})$ nanowires is mostly governed by the shape anisotropy, the magnetization is directed along the wire and differently magnetized domains are separated by head-to-head or tail-totail DWs. These walls were found to have vortex or transverse spin structure depending on the dimensions of the wire [6,7]. Whilst their static properties are well known and numerical simulations of the domain structure are in good agreement with experiment, the dynamic properties of DWs under the influence of spin-polarized currents or external fields have not been completely revealed.

Theoretical calculations and numerical simulations of the field- or current-induced propagation of DWs in permalloy nanowires have shown that there are two types of DW motion.
For small fields or currents the motion is characterized by a DW velocity, which increases linearly with the amplitude of the driving field or current. Above a critical (Walker) threshold the DW speed is drastically reduced and only for much higher fields does it start to increase again with increasing field or current. This is attributed to periodic transformations of the DW structure from vortex to transverse and vice versa $[3,8]$. This general behaviour has been observed experimentally and first evidence of the predicted transformations has been found [9-11].

However, all the measurement techniques that have been used so far either use averaging (e.g. in real-time resistance [12] or magneto-optic Kerr effect (MOKE) measurements [10]) or employ static imaging of the magnetic structure (e.g. magnetic force microscopy (MFM) [13] or X-ray magnetic circular dichroism photoemission electron microscopy (XMCDPEEM) [11]), where the velocity is calculated from the distance the wall travels between two images. This means that possible variations of the DW velocity from shot to shot, which is necessary to fully understand the stochastic character of the DW motion that is induced by thermal fluctuations, randomly distributed pinning centres or the edge roughness of the wire [14], cannot be revealed. To observe stochastic behaviour, 
(a)

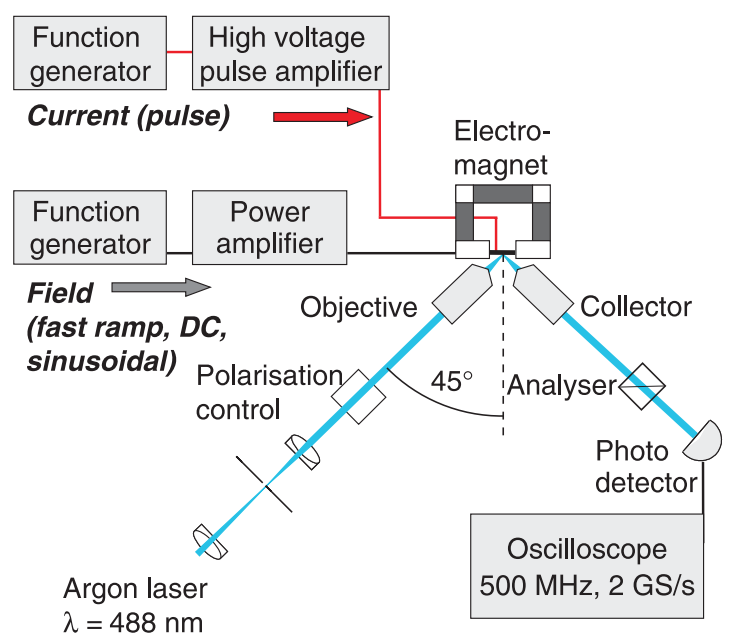

(b)

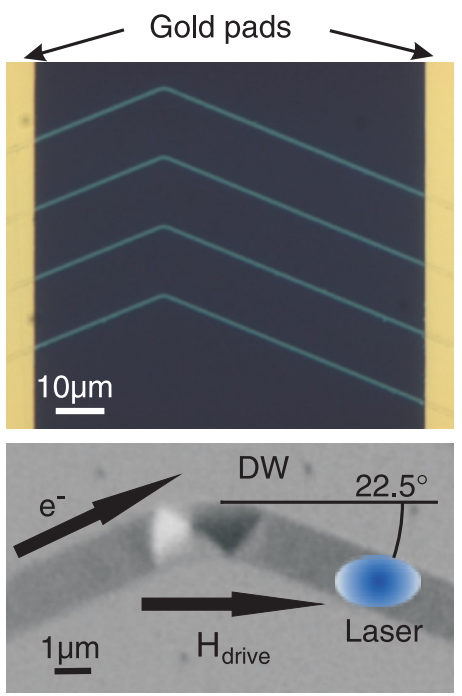

Figure 1. (a) Schematic of the MOKE setup, (b) optical microscope image of $500 \mathrm{~nm}$ wide Py wires including gold contact pads on both sides and $(c)$ XMCD-PEEM image of a DW initialized at a kink in the wire (1500 nm wide).

real-time single shot techniques that probe individual DWs are required, and this is of key importance since future applications will be based on single DW events and not on their average behaviour.

In this paper a focused MOKE setup with a time resolution of $\sim 2 \mathrm{~ns}$ and a spatial resolution of $\sim 1 \mu \mathrm{m}$, capable of realtime single shot measurements is presented. It is shown that the velocity of field-driven DWs in a Py nanowire measured at a single location can vary from shot to shot and that the DWs prepared in identical ways randomly depin at three different external magnetic fields in the samples studied.

\section{Experimental setup}

The MOKE setup, depicted in figure 1(a), consists at its heart of two identical commercial microscope objective lenses of $20 \mathrm{~mm}$ focal length. These focus the beam of a single mode continuous wave $(\mathrm{cw})$ argon ion laser $(488 \mathrm{~nm})$ under an angle of $45^{\circ}$ to an elliptical spot (major axis $\sim 1 \mu \mathrm{m}$ ) on the sample and collect the reflected light. The incoming laser beam, with a power of up to $0.4 \mathrm{~W}$, passes through a beam expander and spatial filter to optimally backfill the objective lens. This relatively high laser power focused to a spot of about $1 \mu \mathrm{m}^{2}$ leads to a high local photon flux, which is needed to achieve a sufficient signal-to-noise ratio for single shot measurements, but results in parasitic heating. Following [15], the temperature increases very locally in the area of the laser spot by about $10 \mathrm{~K}$ for thin Py wires on Si. The thermalization time after the beginning of the illumination is under $100 \mathrm{~ns}$ as determined from time-resolved reflectivity measurements.

The combination of a multi-order $\lambda / 2$-plate and a polarizer ensures a high degree of polarization of the incoming beam at any chosen angle. The measurements shown later on in this paper were done with s-polarized light. The light reflected from the sample is sent through a $\lambda / 4$ plate to restore linear polarization and then through a polarizer which is set to a few degrees from extinction for highest sensitivity [16]. For differential measurements a second polarizer and photodiode together with a non-polarizing beam splitter can be installed. The transmitted light is detected by a fast ( $<2 \mathrm{~ns}$ rise time) custom built photodetector based on a FND-100 (EG\&G) PIN-photodiode, which is biased with $16 \mathrm{~V}$, and the photocurrent is converted to a signal voltage by a $50 \Omega$ load resistor. This signal voltage is amplified by a $2 \mathrm{GHz}$ custom built low noise amplifier and recorded by a $500 \mathrm{MHz}$ and 2 Gsamples s ${ }^{-1}$ Agilent or $4 \mathrm{GHz}$ LeCroy oscilloscope. The risetime of $2 \mathrm{~ns}$ of this detection setup was checked by measuring its response to a femtosecond laser pulse.

The samples investigated (figure $1(b)$ and $(c)$ ) consist of zig-zag shaped Py $\left(\mathrm{Ni}_{80} \mathrm{Fe}_{20}\right)$ wires with a length of about $80 \mu \mathrm{m}$, a width of $1500 \mathrm{~nm}$ down to $400 \mathrm{~nm}$, a thickness of 20-25 nm and an angle of $22.5^{\circ}$ to the plane of incidence as shown in figure $1(c)$. The wires were fabricated by electronbeam lithography, sputter or molecular beam epitaxy (MBE) deposition on a Si substrate and subsequent lift-off. They are electrically contacted with gold pads on either side of the wire. The sample together with the magnet assembly can be positioned relative to the laser spot using a two axis linear motion stage with high-precision actuators (Newport). The actuators include optical sensors that allow closed-loop control of the sample position and permit raster scans with sub- $100 \mathrm{~nm}$ precision. A backlash compensation is included to minimize mechanical hysteresis. For the initialization of the sample a saturating magnetic field is applied and removed in the plane of the sample and perpendicular to the plane of incidence. After reducing the field to zero, DWs are generated at the corners (kinks) in the wires [17]. Another pair of coils producing a magnetic field $H_{\text {drive }}$ in the plane of incidence can then be used to propagate the DWs. This field can be ramped up to $50 \mathrm{G}$ in $50 \mu \mathrm{s}$, varied sinusoidally in time or simply take a constant value, e.g. for field-assisted current-induced motion of the DWs. For current-induced motion of DWs, current pulses of about $10 \mathrm{~ns}$ rise time and selected shape can be injected with a $80 \mathrm{MHz}$ pulse generator and a custom made fast high voltage amplifier. 


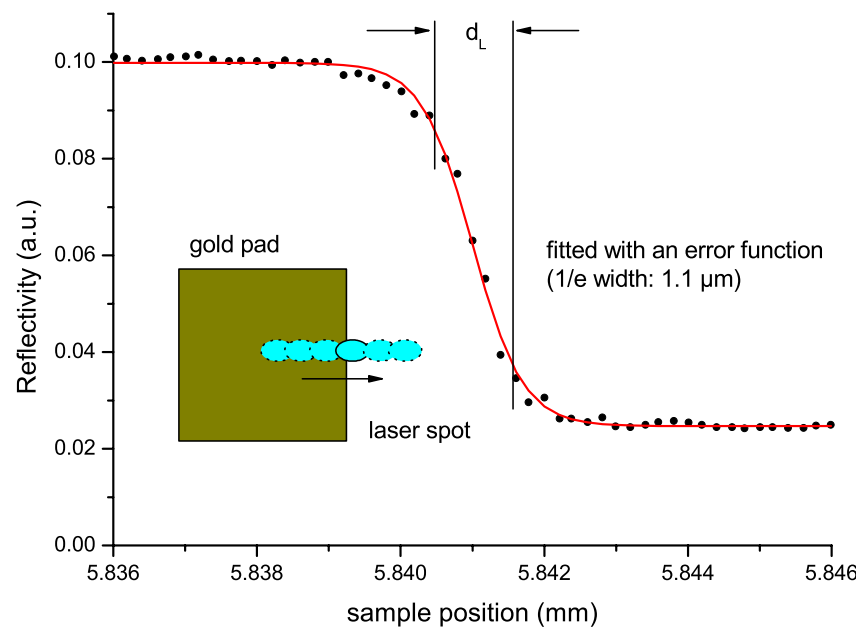

Figure 2. Reflectivity trace obtained by scanning the focused MOKE laser spot over the edge of a gold contact pad onto the Si substrate. Fitting with an error function gives the size of the laser spot.

\section{Method}

For MOKE measurements the laser is focused and the size of the laser spot is determined by scanning over the edge of a gold contact pad onto the substrate, which has a different reflectivity from the pad. The size of the laser spot $d_{\mathrm{L}}$ can then be calculated by fitting the trace with an error function, as shown in figure 2 . Once a diameter of about $1 \mu \mathrm{m}$ has been obtained the laser spot is moved to a position of interest on a wire. DWs are prepared at the kinks as described above and the wall is driven through the laser spot by a field ramp or current pulse. This results in a change in magnetization within the laser spot and hence a change in light intensity reaching the photo detector. A typical trace can be seen in figure $3(a)$. For comparison a trace without a DW prepared is shown in figure $3(b)$.

To reduce the heat load, the sample is only illuminated some $\mu$ s before the start until the end of the current pulse or field ramp, using an electro-optical modulator (EOM) and polarizer as a shutter in the laser beam. Some low frequency noise comes from the EOM and the photodiode, but it is the same for every shot. By averaging over a large number of shots without a DW a reference curve can be obtained with which the data trace can be normalized (figure 4). To reduce the influence of artefacts coming from signal processing the field ramp or current pulse is delayed with respect to the beginning of the illumination and therefore the depinning event shifted to a point about $25-35 \mu$ s away from the beginning of the illumination where the noise has settled. The jump in the intensity which remains after normalization corresponds to the change of magnetization and incorporates two pieces of information. One is the time $t_{0}$ at which the DW traverses the laser spot and the other is the width of the transition $\Delta T$. When the laser spot is positioned sufficiently close to a kink and the field is ramped up linearly, the time $t_{0}$ is a measure of the pinning strength of the DW at the kink, since the dependence of the external field on time is known and the slope of the magnetic
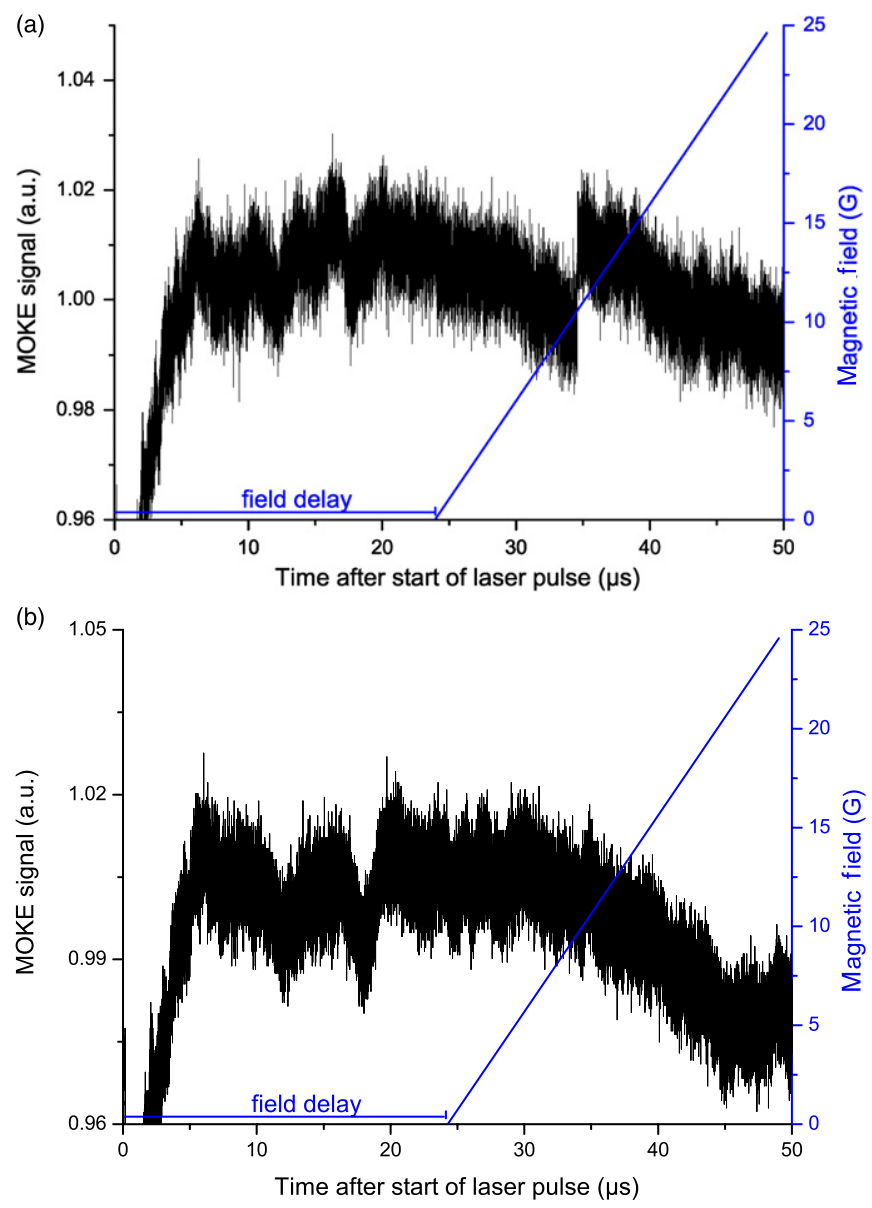

Figure 3. (a) Raw data (MOKE signal) showing a DW traversing the MOKE laser spot at approximately $35 \mu$ s after the start of the laser pulse indicated by a sharp jump in the signal. The slow rise at the beginning is due to the laser light being switched on. (b) For comparison raw data without a DW prepared. No jump is visible.

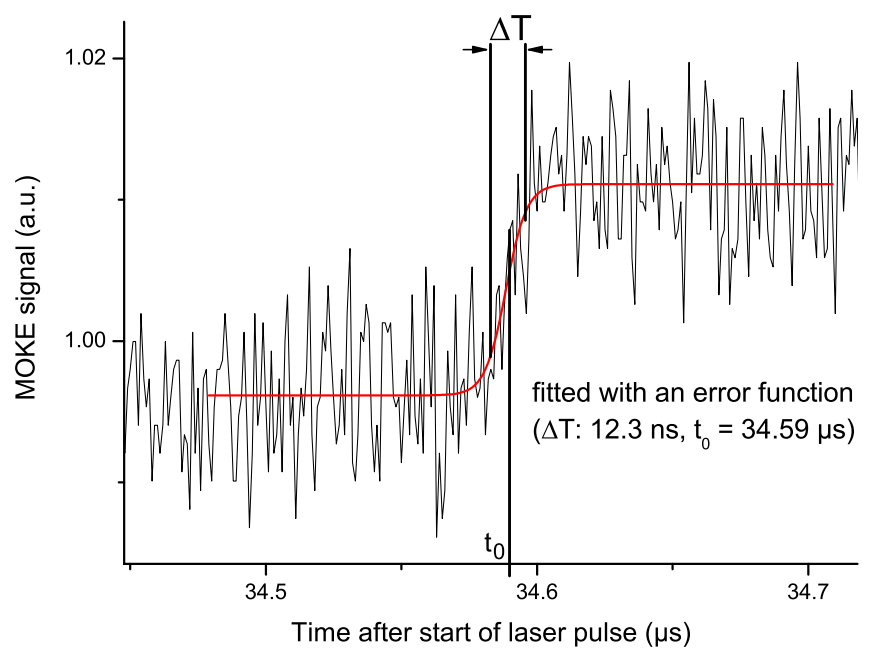

Figure 4. Background corrected and normalized MOKE data trace fitted with an error function. The DW crosses the centre of the laser spot at $t_{0}=34.59 \mu \mathrm{s}$.

field is slow compared with the DW velocity. In currentinduced experiments, where it can be assumed that the DW starts moving at the time the current starts flowing $(t=0)$, the time $t_{0}$ is a measure of the average velocity of the DW. 

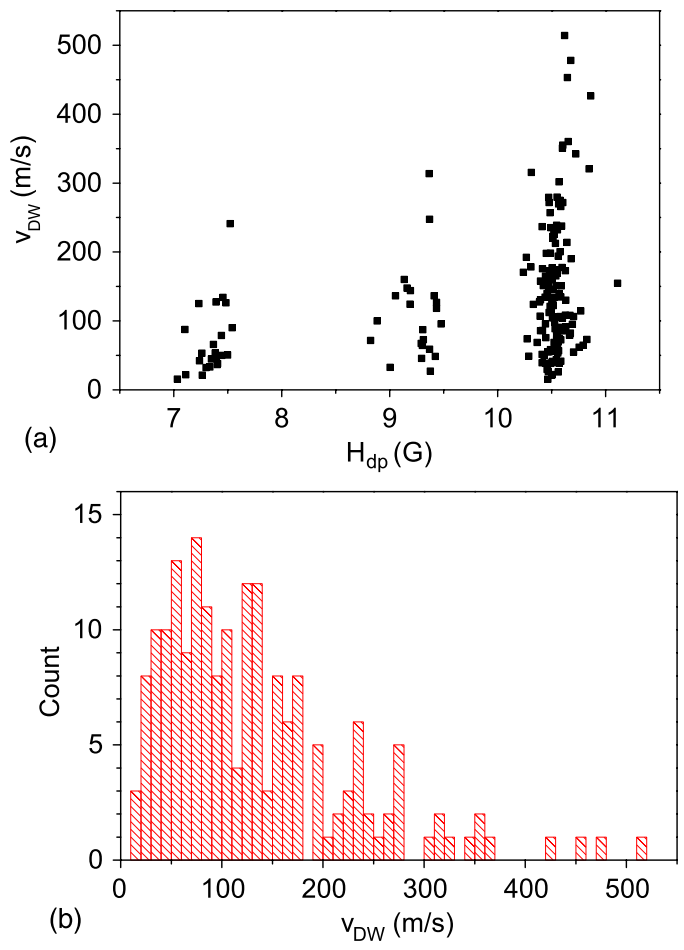

Figure 5. (a) DW velocity $v_{\mathrm{DW}}$ versus depinning field $H_{\mathrm{dp}}$ and (b) probability distribution of $v_{\mathrm{DW}}$ in a $1500 \mathrm{~nm}$ wide Py wire $10 \mu \mathrm{m}$ away from the DW start position.

In contrast, the transition time $\Delta T$ is a direct local measure of the speed of the DW. Assuming the DW has a width of $w_{\mathrm{DW}}$, the speed of the DW is given by:

$$
v_{\mathrm{DW}}=\frac{w_{\mathrm{DW}}+d_{\mathrm{L}}^{\prime}}{\Delta T}
$$

with the effective spot size $d_{\mathrm{L}}^{\prime}=\left(d_{\mathrm{L}} / \sqrt{2-\cos ^{2}\left(22.5^{\circ}\right)}\right) \approx$ $0.93 d_{\mathrm{L}}$ to account for the tilted geometry and the elliptical shape of the spot. If $w_{\mathrm{DW}}=1 \mu \mathrm{m}$ and $d_{\mathrm{L}}=1 \mu \mathrm{m}$ are assumed, the maximum detectable velocity is $1000 \mathrm{~m} \mathrm{~s}^{-1}$. Distributions of $v_{\mathrm{DW}}$ and the pinning field can then be obtained by repeatedly preparing and propagating DWs through the laser spot.

\section{Results}

Results obtained from a $1500 \mathrm{~nm}$ wide and $25 \mathrm{~nm}$ thick wire with the laser focused at a position $10 \mu \mathrm{m}$ away from the starting point of the DWs are presented here. DWs, which at the chosen width and thickness of the wire have a vortex spin structure [7], were repeatedly prepared at the kinks in the wire, the field ramped from $0 \mathrm{G}$ with a speed of $1 \mathrm{G} \mu \mathrm{s}^{-1}$ and the times $t_{0}$ and $\Delta T$ were measured. As the field ramps linearly with time, $t_{0}$ can be directly converted to the corresponding pinning field, and the velocity $v_{\mathrm{DW}}$ is calculated by assuming a DW width of $1 \mu \mathrm{m}$. The data from 200 shots are displayed in the histograms in figure 5.

The depinning field $H_{\mathrm{dp}}$ shown in figure 5(a) exhibits three distinct accumulations of data around 7.5, 9 and $10.5 \mathrm{G}$, which is an indication of the stochastic character of the depinning process, which can happen in different channels.
In each depinning event one of these channels connected to one of the observed depinning fields is chosen with a different probability. The first two depinning channels at 7.5 and $9 \mathrm{G}$ have about the same probability and the last at $10.5 \mathrm{G}$ has a six times larger probability, being chosen by three quarters of the DWs. The different channels could form because of slight inhomogeneities or edge roughnesses of the wire forming a potential landscape the DW has to travel through. The described accumulation of the depinning at more than one depinning field was also reproducibly observed at different positions of the same sample and on other samples, too. An influence on the DW of the power deposited by the laser being switched on during depinning is not probable due to the distance between the laser spot and the starting point $(10 \mu \mathrm{m})$ and the very local heating of the laser. In spintronic devices utilizing DW motion, highly reproducible switching may be necessary and thus it is essential to understand and control the stochastic depinning of the DWs.

Taking a single value of $H_{\mathrm{dp}}$, e.g. $7.5 \mathrm{G}$, there is a wide distribution of velocities. Moving to 9 and $10.5 \mathrm{G}$ the average velocity and the width of the velocity distribution increase, because the DWs, having depinned later, subsequently travel at a higher field. Figure 5(b) shows a detailed probability distribution of the DW velocities. The distribution is skewed towards lower velocities: it extends from $10 \mathrm{~m} \mathrm{~s}^{-1}$ to more than $500 \mathrm{~m} \mathrm{~s}^{-1}$ and peaks at $\sim 80 \mathrm{~m} \mathrm{~s}^{-1}$. The most probable measured DW velocity is in good agreement with previous experiments $[9,10]$, which average over several thousand single events. A more systematic study of the width of this distribution which may be influenced by the variation of DW speeds, the changing DW width and the noise level of the experiment itself is beyond the scope of this paper and will be discussed elsewhere.

\section{Conclusion}

In conclusion, we have presented the details of a single shot focused MOKE magnetometer for capturing individual shot DW measurements of motion in Py nanowires. The instrument has a time resolution better than $2 \mathrm{~ns}$ and a spatial resolution of $\sim 1 \mu \mathrm{m}$. First measurements of the field-induced DW velocity distribution at a spot $10 \mu \mathrm{m}$ away from the DW starting position were made. An accumulation of DW depinning at three distinct depinning fields was observed showing the stochasticity of the process. The measured DW velocity varied significantly between 10 and a few $100 \mathrm{~m} \mathrm{~s}^{-1}$ with a most probable velocity of $\sim 80 \mathrm{~m} \mathrm{~s}^{-1}$.

\section{Acknowledgments}

The authors acknowledge support from the Deutsche Forschungsgemeinschaft (SPP 1133 and SFB 513), the European Spinswitch Research Training Network and the EU (Human Resources and Mobility Programme). PM is grateful for financial support from the Studienförderwerk Klaus Murmann of the Stiftung der Deutschen Wirtschaft. 


\section{References}

[1] Thiaville A, Nakatani Y, Miltat J and Suzuki Y 2005 Micromagnetic understanding of current-driven domain wall motion in patterned nanowires Europhys. Lett. 69990

[2] He J, Li Z and Zhang S 2006 Current-driven vortex domain wall dynamics by micromagnetic simulations Phys. Rev. B 73184408

[3] Mougin A, Cormier M, Adam J P, Metaxas P J and Ferr J 2007 Domain wall mobility, stability and walker breakdown in magnetic nanowires Europhys. Lett. 7857007

[4] Parkin S S P 2004-2007 US Patent no. 6834005, 6898132, 7031178, 723686,

[5] Allwood D A, Xiong G, Faulkner C C, Atkinson D, Petit D and Cowburn R P 2005 Magnetic domain-wall logic Science 3091688

[6] Kläui M, Vaz C A F, Bland J A C, Heyderman L J, Nolting F, Pavlovska A, Bauer E, Cherifi S, Heun S and Locatelli A 2004 Head-to-head domain-wall phase diagram in mesoscopic ring magnets Appl. Phys. Lett. 85 5637-9

[7] Laufenberg M et al 2006 Observation of thermally activated domain wall transformations Appl. Phys. Lett. 88052507

[8] Lee J-Y, Lee K-S, Choi S, Guslienko K Y and Kim S-K 2007 Dynamic transformations of the internal structure of a moving domain wall in magnetic nanostripes Phys. Rev. B 76184408

[9] Hayashi M, Thomas L, Rettner C, Moriya R and Parkin S S P 2007 Direct observation of the coherent precession of magnetic domain walls propagating along permalloy nanowires Nature Phys. 3 21-5
[10] Yang J, Nistor C, Beach G S D and Erskine J L 2008 Magnetic domain-wall velocity oscillations in permalloy nanowires Phys. Rev. B 77014413

[11] Heyne L et al 2008 Relationship between nonadiabaticity and damping in permalloy studied by current induced spin structure transformations Phys. Rev. Lett. 100066603

[12] Hayashi M, Thomas L, Bazaliy Ya B, Rettner C, Moriya R, Jiang X and Parkin S S P 2006 Influence of current on field-driven domain wall motion in permalloy nanowires from time resolved measurements of anisotropic magnetoresistance Phys. Rev. Lett. 96197207

[13] Yamaguchi A, Ono T, Nasu S, Miyake K, Mibu K and Shinjo T 2004 Real-space observation of current-driven domain wall motion in submicron magnetic wires Phys. Rev. Lett. 92077205

[14] Martinez E, Lopez-Diaz L, Torres L, Tristan C and Alejos O 2007 Thermal effects in domain wall motion: micromagnetic simulations and analytical model Phys. Rev. B 75174409

[15] Nistor C, Beach G S D and Erskine J L 2006 Versatile magneto-optic Kerr effect polarimeter for studies of domain-wall dynamics in magnetic nanostructures Rev. Sci. Instrum. 77103901

[16] Cantwell P R, Gibson U J, Allwood D A and Macleod H A M 2006 Optical coatings for improved contrast in longitudinal magneto-optic Kerr effect measurements J. Appl. Phys. 100093910

[17] Kläui M, Jubert P-O, Allenspach R, Bischof A, Bland J A C, Faini G, Rüdiger U, Vaz C A F, Vila L and Vouille C 2005 Direct observation of domain-wall configurations transformed by spin currents Phys. Rev. Lett. 95026601 